\title{
TUMOR FIBROSO SOLITÁRIO DO RIM: RELATO DE CASO
}

\author{
Rogério Saint-Clair Pimentel Mafra* \\ Rafael Morroni de Oliveira* \\ Arthur Guilherme Lobato de Castro ${ }^{*+*}$ \\ Luiz Ronaldo Alberti
}

\begin{abstract}
RESUMO: Trata-se de um caso de um raro tipo de câncer em rim esquerdo de um paciente do sexo masculino, com 63 anos de idade, admitido no serviço e que foi submetido a uma propedêutica minuciosa, incluindo exames de imagem de ultrassom e ressonância nuclear magnética, por queixas de dor lombar esquerda, microhematúria e sintomas francos de prostatismo e disfagia progressiva. Os achados ultrassonográficos e radiológicos da massa encontrada sugeriram 0 diagnóstico de um tumor maligno, de origem parenquimatosa. Os estudos anátomopatológico e imuno-histoquímico da peça ressecada, no entanto, diagnosticaram uma variante benigna do raro tumor fibroso solitário, originário do polo inferior do rim esquerdo.
\end{abstract}

PALAVRAS-CHAVE: Tumor Fibroso Solitário; Neoplasias Renais; Carcinoma de Células Renais.

\section{SINGLE FIBROUS TUMOR IN THE KIDNEY: A CASE REPORT}

\begin{abstract}
A rare type of cancer in the left kidney of a 63-year-old male is analyzed. The patient was submitted to a detailed propaedeutic survey comprising ultra-sound and magnetic nuclear resonance images due to complains of pain on the left lumbar side, micro-hematuria and symptoms of prostate infection and progressive dysphagia. Ultrasound and radiological tests suggested a parenchymal malign tumor. Anatomical, pathological and immunohistochemical studies of the dried section diagnosed a benign variant of a rare single fibrous tumor derived from the lower section of the left kidney.
\end{abstract}

KEYWORDS: Single Fibrous Tumor; Kidney Cancer; Carcinomia of Kidney Cells.

\section{INTRODUÇÃO}

0 tumor fibroso, ou mesotelioma fibroso, foi inicialmente descrito por Klemperer e Rabin (1931) como uma neoplasia de células fusiformes rara e de origem mesotelial e comportamento biológico variável. Constituem neoplasias de partes moles, descrito mais comumente na cavidade toráxica, mais precisamente na pleura (TEIXEIRA et al., 2014). Os tumores fibrosos solitários são neoplasias que frequentemente exibem um padrão vascular de hemangiopericitoma. 0 acometimento renal é raríssimo (CUELLO; BRUGES, 2013; SFOUNGARISTOS et al., 2012; MARTINO; BÖHM; KLATTE, 2012). Os métodos de imagens utilizados atualmente apenas sugerem a possibilidade de malignidade do tumor, pois não há características de imagem capazes de definir o potencial de benignidade ou malignidade dessa neoplasia. Portanto, 0 achado macroscópico e a análise histológica e imunohistoquímica da lesão são cruciais (ABDULLAH et al., 2013; WANG et al., 2014; ZONG et al., 2012).

\footnotetext{
"Doutor em Medicina; Docente titular da Faculdade de Medicina (FAMINAS BH); Pesquisador CNPq

** Docente adjunto da disciplina de Urologia da Universidade Estadual de Montes Claros (MG), Brasil.

*** Discente de medicina pela Faculdade de Minas/FAMINAS-BH, Belo Horizonte (MG), Brasil; E-mail: arthurguilherme5.0@hotmail.com

${ }^{*}$ Docente adjunto do Departamento de Cirurgia da Universidade Federal de Minas Gerais (UFMG); Docente pesquisador da Santa Casa de BH; Pesquisador 2 do CNPq.
} 
Pela escassez do tema na literatura, ainda não há um consenso no acompanhamento dos pacientes após o tratamento cirúrgico, mas, considerando 0 comportamento clínico duvidoso, podemos associar a variante benigna do tumor a uma neoplasia maligna metastática agressiva em potencial. Portanto, é sempre recomendado, quando possível, o tratamento cirúrgico (WANG et al., 2014; ZONG et al., 2012).

\section{METODOLOGIA}

Foi realizada revisão bibliográfica sobre tumor solitário fibroso do rim nas bases de dados Pubmed, LiLACS e Scielo com os seguintes termos: "solitary fibrous tumor" kidney neoplasia", e "kidney cancer". Foram selecionados e revisados os artigos de maior relevância correspondente ao período dos anos entre 1931 e 2015. Foi realizada a descrição do caso clínico, e, para a citação bibliográfica, foi utilizado o software Endnote X7 for Mac. Foram seguidas todas as normas de pesquisa de acordo a legislação vigente de acordo com o CAAE: 55548116.3.0000.5138, e número do comprovante: 036026/2016, pela instituição proponente citada: Instituto de Ensino e Pesquisa da Santa Casa de Belo Horizonte.

\section{RELATO DE CASO}

Trata-se de um paciente de 63 anos, fazendeiro, admitido pelo Hospital Santa Casa de Belo Horizonte - MG, com relato de disfagia progressiva para alimentos sólidos, emagrecimento não quantificado de seis meses de evolução, dor de moderada intensidade em região lombar de início há quatro meses e sintomas urinários obstrutivos como jato fraco, hesitação, intermitência, sensação de esvaziamento vesical incompleto e noctúria. Seus antecedentes mórbidos se resumiam à hipertensão arterial sistêmica, controlada com uso regular de losartan de $50 \mathrm{mg}$. Ao exame inical, seu estado geral se encontrava comprometido, hipocorado, alerta, hidratado no limiar, anictérico e acianótico ao exame clínico. Avaliação cardiorrespiratória sem alterações. A avaliação abdominal revelou palpação profunda dolorosa do flanco esquerdo, sem evidência de massas. Durante 0 exame de toque retal, a próstata evidenciou peso de $80 \mathrm{~g}$, com consistência fibroelástica e ausência de nódulos palpáveis.

Os exames laboratoriais mostraram anemia importante (hemoblobina $7,2 \mathrm{~g} / \mathrm{dL}$ ), alteração da função renal (creatinina 3,2 $\mathrm{mg} / \mathrm{dL}$ ) e sedimento na urina revelou hematúria microscópica. Esôfagograma revelou Megaesôfago Grupo III de Rezende (Figura 1). Ultrassonografia do aparelho urinário descreveu a presença de uma massa renal complexa em topografia do rim esquerdo, afilamento do parênquima renal à direita com ureterohidronefrose bilateral, sendo esta maior à direita, além de espessamento importante de parede vesical.

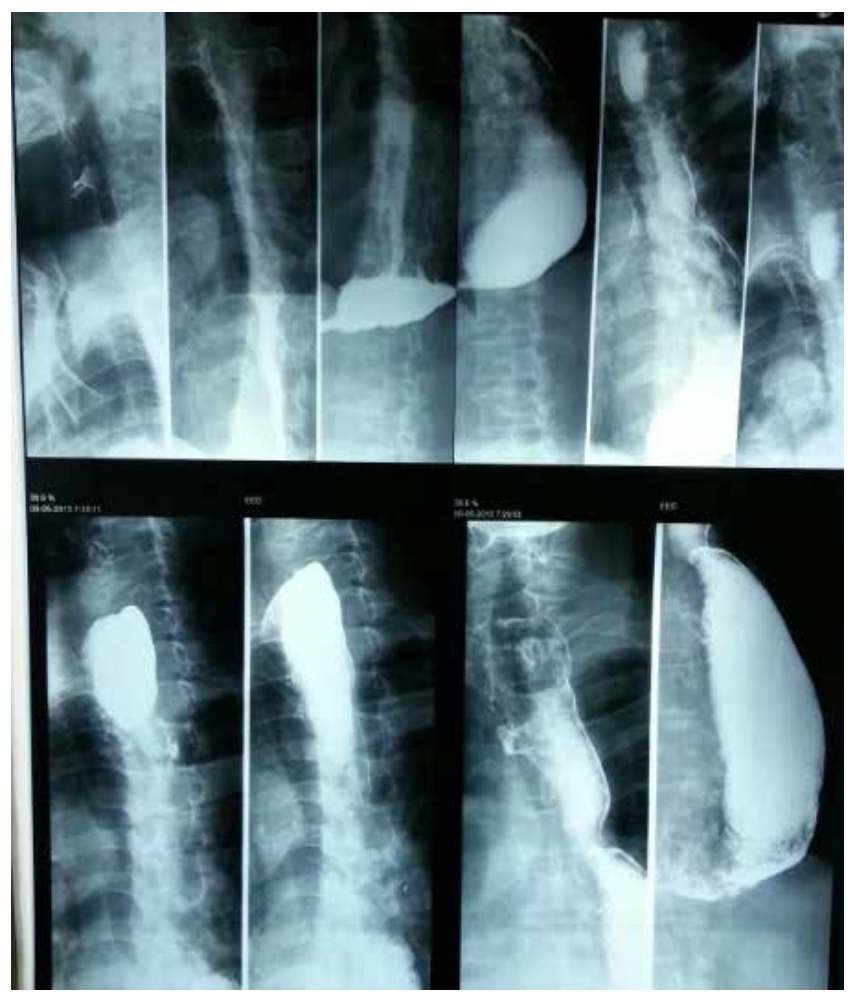

Figura 1. Megaesôfago Grau III. Observe dilatação importante com grande retenção de contraste e afilamento distal

A uretrocistografia miccional complementar confirmou bexiga de esforço, com múltiplos divertículos vesicais, refluxo vesicoureteral grau $\mathrm{V}$ e obstrução importante ao fluxo no nível da uretra prostática (Figura 2). 


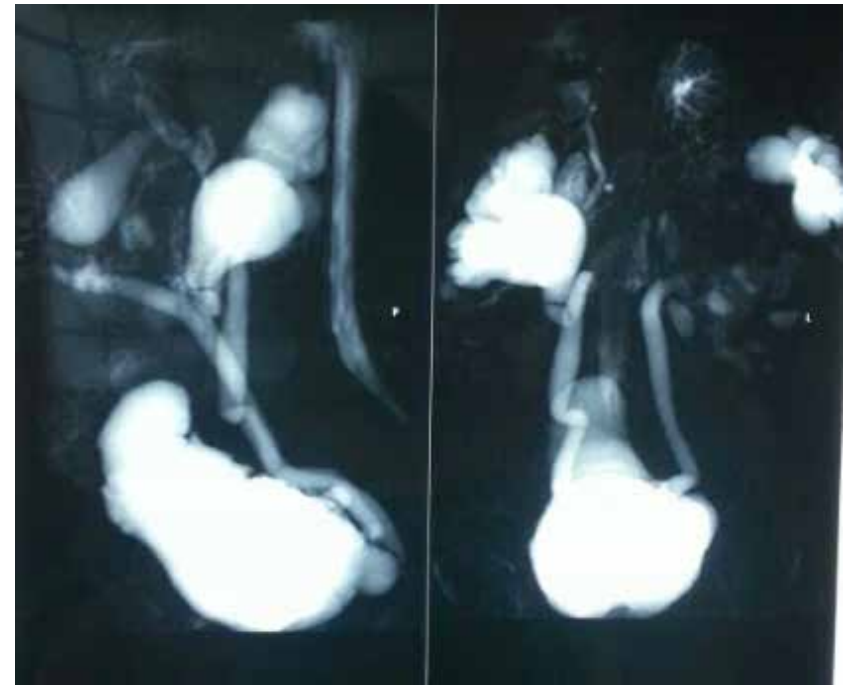

Figura 2. Uretrocistografia: observe refluxo vesicoureteral severo com bexiga em aspecto de "árvore de Natal"

0 exame de Ressonância Nuclear Magnética (RNM) de abdome e pelve revelou volumosa massa renal à esquerda, com íntima relação com 0 polo inferior, mostrando captações heterogêneas do contraste gadolíneo, sugestivas de necrose, bem como calcificações internas (Figuras 3 e 4).

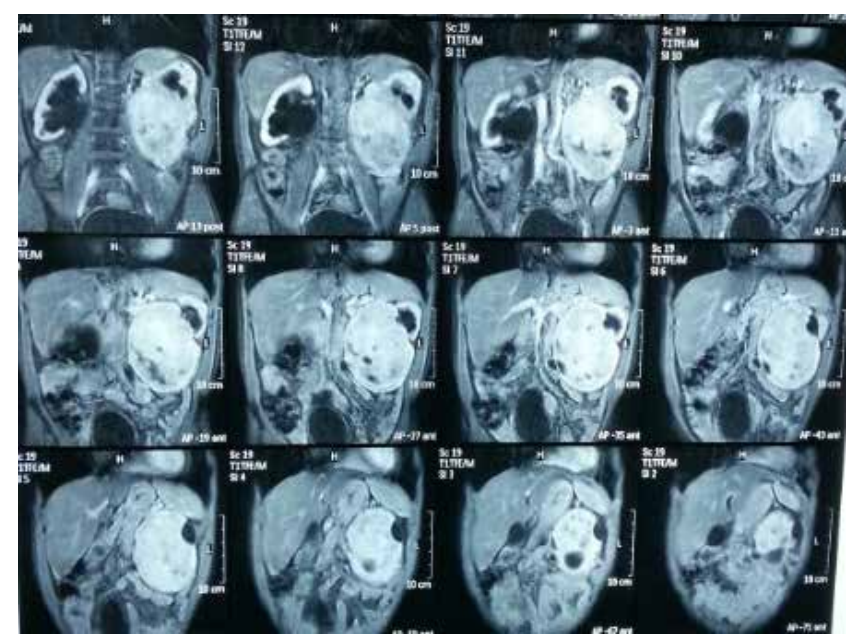

Figura 3. RNM Corte coronal - observe volumosa lesão espansiva estendendo-se até a pelve, rechaçando pâncreas superiormente, e grandes vasos medialmente

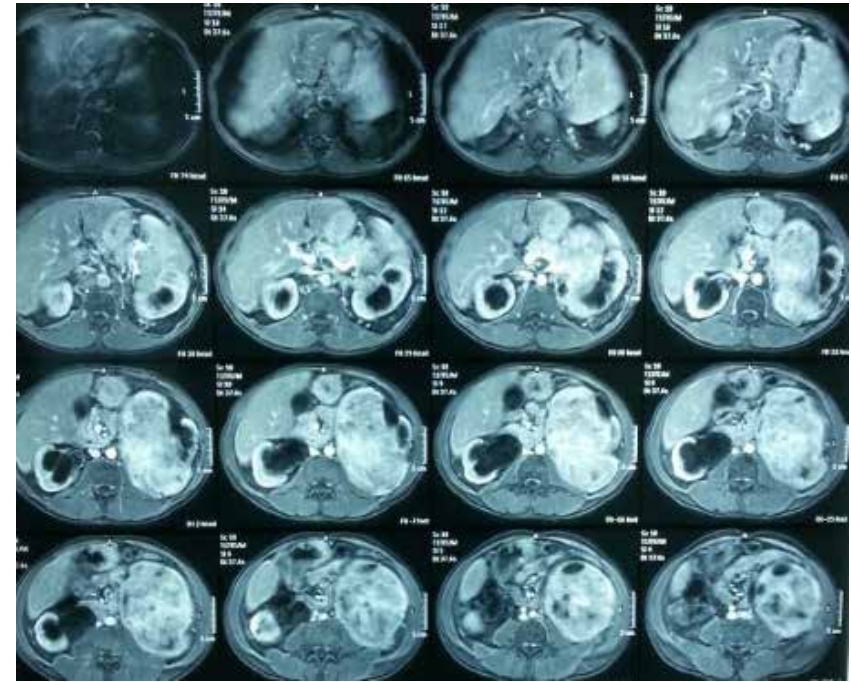

Figura 4. RNM Corte axial. Observe hidronefrose concomitante no rim direito, ausência de plano de clivagem do rim esquerdo com o pâncreas e captação heterogênea com impressão de áreas de necrose em permeio no tumor

A hipótese de uma neoplasia maligna renal foi interrogada, e, com base nessa suspeita, optamos por realizar nefrectomia radical esquerda por via subcostal e linfadenectomia perihilar. A cirurgia transcorreu sem complicações. A peça cirúrgica foi enviada para 0 laboratório de Anatomia Patológica do mesmo serviço (Santa Casa BH).

Ao exame macroscópico, a peça cirúrgica foi composta pelo rim esquerdo e gordura perirenal medindo $18 \mathrm{~cm}$ de comprimento, $14 \mathrm{~cm}$ de altura, 9 cmde largura e peso de $1.350 \mathrm{~g}$.

Macroscopicamente, a peça apresentou múltiplas projeções nodulares na superfície exterior. 0 exame microscópico de cortes histológicos, utilizando coloração de rotina $(\mathrm{HE})$, mostrou neoplasia caracterizada por proliferação de células ovoides, fusiformes, ligeiramente alongadas e sem atipia, com núcleos discretos produzindo massas sólidas com deposição de colágeno intercalada com tecido estromal dentro de estroma fibroso. Foram observados vasos ectasiados tortuosos, semelhante ao padrão de hemangiopericitoma (Figura 5). 


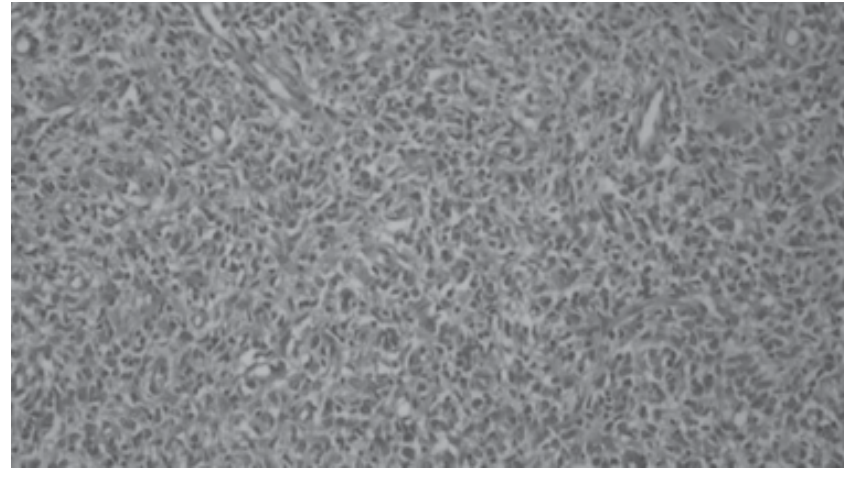

Figura 5. Microscopia mostrou proliferação anômala de células rodeadas por um estroma fibroso e colágeno em permeio

Figuras mitóticas ocasionais foram vistas (menos que 03 mitoses por 10 campos de alta potência) (Figura 6). Vasos hilares, ureteres e gânglios linfáticos isolados não foram afetados pelo tumor.

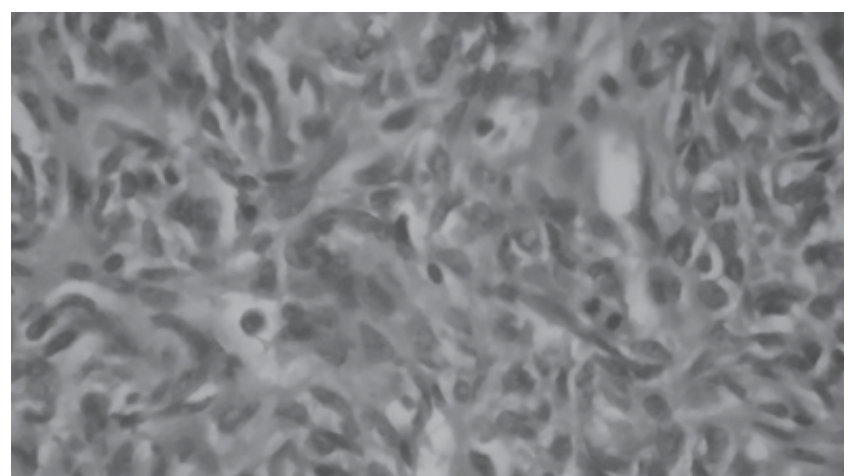

Figura 6. Histologia mostra perda da arquitetura celular com padrão de hialinização perivascular. Deposição de colágeno é frequentemente observada

0 estudo imunohistoquímico revelou negatividade para actina muscular, antígeno GP100, desmina, proteína S-100, proteína MDM2 IF2; e expressão focal para antiCD 34 (células hematopoéticas).

No pós-operatório, o paciente evoluiu com acidose tubular renal tipo IV. Durante a mesma internação, o paciente foi avaliado pela equipe de cirurgia geral que orientou tratamento conservador para o quadro de megaesôfago.

A alta ocorreu após melhora clínica e estabilização da função renal, com sondagem vesical de demora. Algumas semanas depois foi submetido à prostatectomia aberta com reimplante ureteral à direita com válvula antirefluxo.

\section{DISCUSSÃO}

0 tumor fibroso solitário (TFS) pode ser observado em adultos de meia-idade, entre 30 e 80 anos de idade (média de 50 anos), sem predileção por sexo (NAOTO et al., 2014). Foi primeiramente relatado por Klemperer e Rabin em 1931 como um tumor de pleura. ${ }^{1}$ Porém, nos últimos anos, vários relatos de TFS provenientes de outros órgãos foram descritos, incluindo o rim. Sabemos hoje que o tumor pode ser encontrado em qualquer local do corpo (MAGRO et al., 2008).

Clinicamente, o paciente com tumor fibroso solitário no rim pode apresentar massa palpável no flanco ou no abdômen e dor lombar ou desconforto abdominal, associada à compressão local além de hematúria. Os tumores são muitas vezes detectados incidentalmente. Hipoglicemia intermitente pela produção de fator de crescimento Insulina-like também é descrita (NAOTO et al., 2014; MAGRO et al., 2008).

Embora histogênese do TFS permaneça obscura, estudos recentes sugerem fortemente uma origem mesenquimal primitiva ou celular perivascular. A análise macroscópica do TFS mostra áreas bem definidas de massas circunscritas, parcialmente encapsuladas que exibem um crescimento lento e medindo entre 1 e $20 \mathrm{~cm}$ (média de 5-8 cm) (LEROY et al., 2000; WANG et al., 2001).

Achados histopatológicos do TFS incluem perda padrão de arquitetura celular, caracterizada por células fusiformes ou ovoides distribuídas em várias camadas sem arranjo definido, separadas por feixes fibrosos de colágeno hialinizados. 0 padrão de hemangiopericitoma é frequentemente observado (LEROY et al., 2000; WANG et al., 2001).

Tumores atípicos têm arranjos de células fusiformes que contêm citoplasma escasso, padrões mixoides de limites imprecisos, cromatina dispersa em núcleos vesiculares e citoplasma com infiltrado histiocitário. 0 tumor pode infiltrar a parede da veia renal (LEROY et al., 2000; WANG et al., 2001).

Mitoses são escassas, raramente ultrapassando três mitoses por dez campos de alta 
potência (BRUNNERMANN et al., 1999; SOBRADO et al., 1998). Os critérios de diagnóstico para TFS extratorácicas malignas são puramente microscópicos e incluem 0 aumento da celularidade, pleomorfismo e contagem mitótica mais de quatro por dez campos de alta potência (BRUNNERMANN et al., 1999; SOBRAD0 et al., 1998).

0 TFS mostra ampla variedade de padrões de crescimento microscópico. A variante maligna do TFS é composta de lesões hipercelulares que mostram atipias celulares, necrose tumoral, numerosas mitoses (mais de quatro mitoses por dez campos de alta potência) e invasão capsular (WESTRA; GRENKO; EPSTEIN, 2000).

Estudo imunohistoquímico é essencial pelo enorme número de diagnósticos diferenciais, dentre os mais importantes, os fibrossarcomas, leiomiomas e angiomiolipomas. 0 mesmo demonstra intensa positividade difusa das células neoplásicas para marcadores CD34 (90-95\% de casos) e vimentina na maioria dos casos (FUKUNAGA et al., 1997; KOHL; MATHEWS; BAKER, 2006; BOZKURT et al., 2007). Outros marcadores, dentre os quais a proteína S-100, actina músculo-específica (HHF35) e panqueratina (AE1 e AE3), mostram-se negativos na neoplasia (HASEGAWA et al., 1999).

Fatores prognósticos dependem da variante do tumor. A maioria dos casos é benigna, com prognóstico favorável, pois na maioria das vezes não apresentam metástases e não recorrem. Porém seu comportamento biológico ainda é incerto. As metástases podem ocorrer para pulmão, fígado e ossos. Cerca de 10 a $15 \%$ se comportam de forma agressiva, 0 que obriga 0 acompanhamento rigoroso dos pacientes por um longo período (SASAKI et al., 2013).

A excisão cirúrgica completa é 0 tratamento de escolha para esse tipo de lesão. No entanto não há evidência se a nefrectomia parcial tem 0 mesmo resultado da nefrectomia radical (KHATER et al., 2013). Casos de hipoglicemia recorrente podem ser resolvidos com a ressecção cirúrgica (ZHAO; LI; HAN, 2012). 0 acompanhamento pós-operatório em longo prazo é importante, uma vez que o TFS confere comportamento imprevisível e pode recorrer localmente. A forma maligna provoca um curso agressivo da doença, com invasão local, recorrência da doença e metástases. Assim, quimioterapia adjuvante, embora não existam regimes-padrão, é recomendada nos casos de doença maligna, principalmente tumores metastáticos ou não operados.

\section{CONCLUSÃO}

0 TFS é uma neoplasia extremamente incomum e com poucos casos de acometimento renal. Os sintomas dependem do volume do tumor e, no caso do acometimento renal, podem ser escassos e inespecíficos, representados por dor abdominal à palpação profunda e hematúria, como no caso descrito.

As características histológicas da lesão são células de proliferação fusiforme, alongada e oval, com alternância de áreas hipo e hipercelulares, divididas por placas de tecido conjuntivo fibroso. Há ainda proeminência vascular.

0 diagnóstico diferencial pode incluir quaisquer lesões benignas, tais como leiomioma, schwannoma, histiocitoma fibroso benigno, ou lesões malignas, como sarcoma fibromixoide de baixo grau e tumores malignos periféricos da bainha do tendão. Portanto, estudo imuno-histoquímico para investigação diagnóstica da lesão é necessário.

\section{REFERÊNCIAS}

ABDULLAH, D. G. et al. Solitary Fibrous Tumor of the Kidney: A Case Report. Hindawi Publishing Corporation.

Case Reports in Urology. Kayseri, mar. 2013. Disponível em: <http://www.hindawi.com/journals/ criu/2013/147496/>. Acesso em: 10 dez. 2015.

BOZKURT, S. U. etal. Solitary fibrous tumor of the kidney. Acta Pathologica Microbiologica et Immunologica Scadinavica, Lynge, v. 115, n. 3, p. 259-262, mar. 
2007. Disponível em: <http://www.ncbi.nlm.nih.gov/ pubmed/17367473>. Acesso em: 11 dez. 2015.

BRUNNERMANN, R. B. et al. Extrapleural solitary fibrous tumor: a clinicopathologic study of 24 cases. Modern Pathology, v. 12, n. 11, p. 1034-1042, nov. 1999. Disponível em: <http://www.ncbi.nlm.nih.gov/ pubmed/10574600 >. Acesso em: 11 dez. 2015.

CUELLO, J.; BRUGES, R. Malignant solitary fibrous tumor of the kidney: report of the first case managed with interferon. Case Reports in Oncological Medicine. New York, jan. 2013. Disponível em: <http:// www.ncbi.nlm.nih.gov/pmc/articles/PMC3557624/>. Acesso em: 10 dez. 2015.

FUKUNAGA, M. et al. Extrapleural solitary fibrous tumor: a report of seven cases. Modern Pathology, v. 10, n. 5, p. 443-450, maio 1997. Disponível em: <http:// www.ncbi.nlm.nih.gov/pubmed/9160308>. Acesso em: 12 dez. 2015.

HASEGAWA, T. et al. Extrathoracic solitary fibrous tumors: their histological variability and potentially aggressive behavior. Human Pathology, Philadelphia, v. 30, n. 12, p. 1464-1473, dez. 1999. Disponível em: <http://www.ncbi.nlm.nih.gov/pubmed/10667425>. Acesso em: 11 dez. 2015.

KHATER, N. et al. Solitary fibrous tumors of the kidneys: presentation, evaluation, and treatment. Urologia Internationalis, Basel, v. 91, n. 4, p. 373-383, ago. 2013. Disponível em: <http://www.ncbi.nlm.nih.gov/ pubmed/24008397>. Acesso em: 09 dez. 2015.

KLEMPERER, P.; RABIN, C. Primary neoplasm of the pleura: a report of five cases. Archives of Pathology \& Laboratory Medicine. Northfield, 1931.

KOHL, S.; MATHEWS, K.; BAKER, J. Renal hilar mass in an 85-year-old woman. Archives of Pathology \& Laboratory Medicine. Northfield, v. 130, n. 1, p. 117-119, jan. 2006. Disponível em: <http:// www.archivesofpathology.org/doi/10.1043/15432165(2006)130[117:RHMIAY]2.0.C0;2?url_
ver=Z39.88-2003\&rfr_id=ori\%3Arid\%3Acrossref. org\&rfr_dat=cr_pub\%3Dpubmed\&>. Acesso em: 12 dez. 2015.

LEROY, X. et al. Solitary fibrous tumour of the kidney. Urologia Internationalis, Basel, v. 65, n. 1, p. 49-52, 2000. Disponível em: <http://www.ncbi.nlm.nih.gov/ pubmed/10965303 > . Acesso em: 10 dez. 2015.

MAGRO, G. et al. Solitary fibrous tumour of the kidney with sarcomatous overgrowth. Case report and review of the literature. Acta Pathologica Microbiologica et Immunologica Scadinavica. Lynge, v. 116, n. 11, p. 1020-1025, nov.2008. Disponível em: <http://www. ncbi.nlm.nih.gov/pubmed/19133003 > . Acesso em: 09 dez. 2015.

MARTINO, M.; BÖHM, M., KLATTE, T. Malignant solitary fibrous tumour of the kidney: report of a case and cumulative analysis of the literature. Aktuelle Urologie, v. 43, n. 1, p. 59-62, jan. 2012. Disponível em: <http:// www.ncbi.nlm.nih.gov/pubmed/22271338>. Acesso em: 11 dez. 2015.

NAOTO, K. et al. Solitary fibrous tumor of the kidney with focus on clinical and pathobiological aspects. International Journal of Clinical Experimental Pathology, Madison, v. 7, n. 6, maio 2014. Disponível em: <http://www.ncbi.nlm.nih.gov/pmc/articles/ PMC4097243/>. Acesso em: 11 dez. 2015.

SASAKI, H. et al. Distant metastasis from benign solitary fibrous tumor of the kidney. Case Reports of Nephrology and Urology. Basel, v. 3, n. 1, p. 1-8, jan. 2013. Disponível em: <http://www.ncbi.nlm.nih.gov/ pubmed/23466873 >. Acesso em: 11 dez. 2015.

SFOUNGARISTOS, S. et al. Solitary fibrous tumor of the kidney with massive retroperitoneal recurrence. A case presentation. Prague Medical Report, Kateinská, v. 113, n. 3, p. 246-250, 2012. Disponível em: <http:// www.ncbi.nlm.nih.gov/pubmed/22980566>. Acesso em: 11 dez. 2015.

SOBRAD0, C. W. et al. Tumor Fibroso Solitário Retrorretal: relato de caso. Revista Brasileira de 
Coloproctologia, Rio de Janeiro, v. 18, n. 1, p. 37-41, jan./mar. 1998. Disponível em: <http://www.jcol.org. br/pdfs/18_1/07.pdf>. Acesso em: 09 dez. 2015.

TEIXEIRA, U. F. et al. Giant solitary fibrous tumor of retroperitoneum. Revista da Associação Médica do Rio Grande do Sul, Porto Alegre, v. 58, n. 3, p. 237-259, jul./set. 2014. Disponível em: <http://www. amrigs.org.br/revista/58-03/013.pdf>. Acesso em: 10 dez. 2015.

WANG, H. et al. A huge malignant solitary fibrous tumor of kidney: case report and review of the literature. Diagnostic Pathology, London, v. 9, jan. 2014. Disponível em: <http://www.ncbi.nlm.nih.gov/pmc/ articles/PMC3941948/>. Acesso em: 10 dez. 2015.

WANG, J. et al. Large solitary fibrous tumor of the kidney: Report of two cases and review of the literature. The American Journal of Surgical Pathology, Philadelphia, v. 25, n. 9, p. 1194-1199, set. 2001. Disponível em: <http://www.ncbi.nlm.nih. gov/pubmed/11688580 >. Acesso em: 11 dez. 2015.

WESTRA, W. H.; GRENKO, R. T.; EPSTEIN, J. Solitary fibrous tumor of the lower urogenital tract: a report of five cases involving the seminal vesicles, urinary bladder, and prostate. Human Pathology, Philadelphia, v. 31, n. 1, p. 63-68, jan. 2000. Disponível em: <http:// www.ncbi.nlm.nih.gov/pubmed/10665915>. Acesso em: 08 dez. 2015.

ZHAO, G.; LI, G.; HAN, R. Two malignant solitary fibrous tumors in one kidney: Case report and review of the literature. Oncology Letters, Athens, v. 4, n. 5, p. 993995, nov. 2012. Disponível em: <http://www.ncbi.nlm. nih.gov/pmc/articles/PMC3499582/>. Acesso em: 12 dez. 2015.

ZONG, L. et al. Giant solitary fibrous tumor arising from greater omentum. World Journal of Gastroenterology, v. 18, n. 44, p. 6515-6520, nov. 2012. Disponível em: <http://www.ncbi.nlm.nih.gov/pubmed/23197901>. Acesso em: 11 dez. 2015.
Recebido em: 17 de fevereiro de 2016 Aceito em: 11 de maio de 2016 